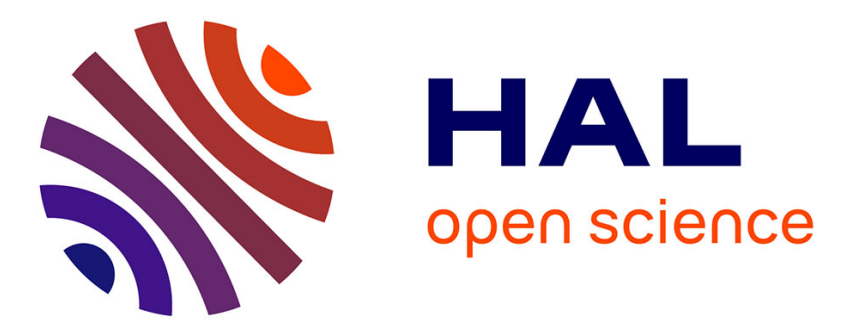

\title{
Polymorphisme chez des souches brésiliennes de l'Hyphomycète entomopathogène Metarhizium anisopliae (Metsch.) Sorokin, inféodées à des Cercopidae
} Guy Riba, Maria Lourdes de Aquino, Sonia Alves Ribeiro

\section{- To cite this version:}

Guy Riba, Maria Lourdes de Aquino, Sonia Alves Ribeiro. Polymorphisme chez des souches brésiliennes de l'Hyphomycète entomopathogène Metarhizium anisopliae (Metsch.) Sorokin, inféodées à des Cercopidae. Agronomie, 1987, 7 (10), pp.763-768. hal-00884951

\section{HAL Id: hal-00884951 https://hal.science/hal-00884951}

Submitted on 1 Jan 1987

HAL is a multi-disciplinary open access archive for the deposit and dissemination of scientific research documents, whether they are published or not. The documents may come from teaching and research institutions in France or abroad, or from public or private research centers.
L'archive ouverte pluridisciplinaire HAL, est destinée au dépôt et à la diffusion de documents scientifiques de niveau recherche, publiés ou non, émanant des établissements d'enseignement et de recherche français ou étrangers, des laboratoires publics ou privés. 


\title{
Polymorphisme chez des souches brésiliennes de l'Hyphomycète entomopathogène Metarhizium anisopliae (Metsch.) Sorokin, inféodées à des Cercopidae
}

Guy RIBA, Maria LOURDES DE AQUINO (*) \& Sonia ALVES RIBEIRO (**)

I.N.R.A., Station de Recherches de Lutte biologique, Centre de Recherches de Versailles, La Minière, F 78280 Guyancourt

(*) Instituto Pernambucano de Agronomia, 50.000 Recife, PE, Brésil

(**) Instituto de Alcohol e Açicar, Carpina, PE, Brésil

RÉSUMÉ

\begin{abstract}
Plusieurs souches brésiliennes de Metarhizium anisopliae, isolées de Cercopides de la canne à sucre ou des pâturages, ont été comparées. Leur agressivité à l'égard de la pyrale du maïs et leurs spectres protéiques obtenus par focalisation isoélectrique de 6 systèmes enzymatiques ont permis de mettre en évidence une grande homogénéité au sein de cette population de souches. Par ces techniques un grand nombre d'isoenzymes sont révélées au sein de chaque souche de cette espèce. Des souches aux profils électrophorétiques différents peuvent cohabiter en un même champ de canne à sucre.

Enfin aucune relation entre la virulence et la composition biochimique n'a pu être décelée.
\end{abstract}

Mots clés additionnels : Mahanarva posticata, isoenzymes, isoélectrofocalisation, virulence.

Genetic variability of Brazilian strains of the entomogenous Hyphomycete Metarhizium anisopliae, isolated from cercopidae.

Several Brazilian strains of Metarhizium anisopliae, isolated from sugar cane or pasture-land cercopids, have been compared. Great homogeneity within the population was found for aggressiveness towards Ostrinia nubilalis and for protein spectra obtained by isoelectric focusing of several enzymes. 34 non-variable isoenzymes were observed among the 27 strains analyzed. On the other hand, in three fields, several different genotypes were simultaneously present. The numerous isoenzymes discovered in each strain demonstrate the great adaptibility of the species. No relationship was observed between virulence and the electrophoretic patterns. Relationships between these data and the parasexuality of $M$. anisopliae are discussed.

Additional key words : Mahanarva posticata, isoenzymes, isoelectric focusing, virulence.

\section{INTRODUCTION}

Depuis plus de 20 ans l'Hyphomycète entomopathogène Metarhizium anisopliae (Metsch.) Sorokin est introduit chaque année dans plusieurs milliers d'hectares de canne à sucre du Nord-Est du Brésil, afin de lutter contre le Cercopidae Mahanarva posticata Stal. (GUAGLiUmi, 1970 ; Veiga et al., 1972). L'incontestable succès de ces applications est pour partie dû à l'évolution concomittante de la population fongique introduite et d'une population endémique autochtone qui affecte régulièrement 10 à 20 p. 100 des insectes. Par ailleurs plusieurs souches de $M$. anisopliae furent isolées au Brésil à partir des Cercopidae des pâturages Deois flavopicta Stal. et Zulia entreriana Berg. (NAves, 1980 ; NAVES \& CORREA, 1982).

Ces divers contextes épidémiologiques d'une même espèce d'entomopathogène inféodée à plusieurs insectes de la même famille constituent des situations de choix pour l'étude du polymorphisme génétique de populations fongiques. L'analyse présentée consiste en la description du polymorphisme isoenzymatique d'un échantillon de souches brésiliennes à l'appui d'une étude précédente (RIBA et al., 1986) qui permit d'identifier, par isoélectrofocalisation, quelques systèmes enzymatiques intracellulaires très polymorphes. Enfin, 
l'étude fut complétée par une évaluation comparative de la virulence des souches à l'égard de larves diapausantes de la pyrale du maïs Ostrinia nubilalis (Hubner), dont on connaît déjà la sensibilité à cette espèce fongique (RIBA et al., 1983).

\section{MATÉRIEL ET MÉTHODES}

\section{A. Le matériel fongique}

Le matériel analysé est constitué de 27 souches monospores brésiliennes de $M$. anisopliae isolées de Cercopidae prélevés en divers contextes agrocoenotiques (tabl. 1). 21 proviennent de cadavres mycosés de $M$. posticata issus de champs de canne à sucre des états de Pernanbouc et Alagoas. Deux d'entre elles, les souches 169 et 170 , sont en fait des isolements effectués dans des champs traités quelques mois auparavant avec une souche déterminée dont on suivra, ainsi, la stabilité des caractères dans un contexte cultural précis. De même la souche 157 , originellement isolée d'adultes de $Z$. entreriana dans un pâturage de l'état du Mato Grosso, fut massivement introduite dans des champs de canne à sucre près de Maceio (Alagoas) d'où provient la souche 155, ou dans des pâturages de Janauba (Minas Gerais) d'où est issue la souche 151. Enfin, les souches $157,158,159$ et 171 prélevées en des régions fort éloignées du Nord-Est du Brésil, continueront à placer la variabilité des populations de $M$. anisopliae du Nord-Est brésilien par rapport à son ampleur dans l'ensemble du pays.

\section{B. La production des conidies}

Les conidiospores sont obtenues après 15 jours de culture des souches sur milieu complet gélosé contenant : $0,36 \mathrm{~g} \mathrm{KH}_{2} \mathrm{PO}_{4} ; 1,42 \mathrm{~g} \mathrm{Na}_{2} \mathrm{HPO}_{4} ; 1 \mathrm{~g} \mathrm{KCl}$; $0,6 \mathrm{~g} \mathrm{MgSO}_{4} 7 \mathrm{H}_{2} \mathrm{O} ; 0,7 \mathrm{~g} \mathrm{NH}_{4} \mathrm{NO}_{3} ; 5 \mathrm{~g}$ extrait de levure ; $10 \mathrm{~g}$ glucose ; $18 \mathrm{~g}$ gélose pour $1000 \mathrm{ml}$ d'eau distillée. Les spores sont récoltées par grattage superficiel du milieu puis mises en suspension dans l'eau distillée.

\section{Les préparations enzymatiques}

Le mycélium est produit en 7 jours de culture profonde agitée $(100 \mathrm{rpm})$ à $25^{\circ} \mathrm{C}$ dans $50 \mathrm{ml}$ de milieu d'ADAMEK (1965) mis dans des erlenmeyers de $150 \mathrm{ml}$. La masse mycélienne est alors filtrée sur mousseline, rincée 4 fois dans de l'eau distillée puis dans du tampon TRIS-EDTA $5 \cdot 10^{-3 \mathrm{M}}$, pH 8,2 , séchée sur papier absorbant, pesée et finalement broyée dans l'azote liquide. L'extrait brut est repris dans le même tampon par centrifugation à $15000 \mathrm{~g}$ pendant $90 \mathrm{mn}$, puis distribué en fraction de $50 \mu \mathrm{l}$ conservées à $-35^{\circ} \mathrm{C}$. La teneur protéique des fractions est estimée par la méthode de LOWry et al. (1951). Pour chaque isolat, 3 extractions sont effectuées, chacune d'elles étant examinée sur des gels différents.

TABLEAU 1

Origine géographique et entomologique des 27 souches brésiliennes de Metarhizium anisopliae.

Entomological and geographical origin of 27 Brazilian Metarhizium anisopliae strains.

\begin{tabular}{|c|c|c|c|c|}
\hline Souches & \multicolumn{2}{|c|}{ Insecte-hôte } & Origine géographique & Année d'isolement \\
\hline 149 & \multicolumn{2}{|c|}{ Mahanarva posticata } & Nord Pernanbouc (Usine Mussurepe) & 1977 \\
\hline 144 & - & - & Nord Pernanbouc (Usine Cruangi) & 1980 \\
\hline 145 & - & -- & Nord Pernanbouc (Usine N. Senhora dos Maravillas) & - \\
\hline 153 & - & - & Nord Ouest Pernanbouc (Carpina) & 1980 \\
\hline 154 & - & - & Nord Ouest Pernanbouc (Carpina) & 1979 \\
\hline 170 & - & - & Parcelle traitée en 1980 avec 154 & 1981 \\
\hline 168 & - & - & $\begin{array}{l}\text { Parcelle traitée en } 1980 \text { avec } 154 \\
\text { (Carpina - Usine Ste Thérésa) }\end{array}$ & 1980 \\
\hline 169 & - & - & Parcelle traitée en 1980 avec 168 & 1981 \\
\hline 147 & - & - & Sud Pernanbouc & 1979 \\
\hline 148 & - & - & Sud Pernanbouc & 1980 \\
\hline 146 & - & - & Sud Pernanbouc (Usine Cucau) & 1981 \\
\hline 150 & - & - & Alagoas (Maceio) & 1977 \\
\hline 152 & - & - & Alagoas (Usine Triumph) & 1981 \\
\hline 164 & - & - & Alagoas (Usine Triumph) & 1981 \\
\hline 161 & - & - & Alagoas (Usine Peixe) & 1981 \\
\hline 162 & - & - & Alagoas (Usine Peixe) & 1980 \\
\hline 163 & - & - & Alagoas (Usine St Michel) & 1981 \\
\hline 165 & - & - & Alagoas (Usine St Michel) & 1981 \\
\hline 156 & - & - & Alagoas (Usine Charles) & 1981 \\
\hline 166 & - & - & Alagoas (Usine Cururipe) & 1981 \\
\hline 167 & - & - & Alagoas (Usine Ste Cécile) & 1981 \\
\hline 171 & - & - & Sao Paolo & 1981 \\
\hline 157 & Zulia & iana & Mato Grosso & 1978 \\
\hline 151 & M. $p$ & & Introduction de 157 dans le Minas Gerais & 1981 \\
\hline 155 & Diatraed & haralis & Introduction de 157 dans l'Alagoas & 1981 \\
\hline 159 & Deois & pleta & Brasilia & 1979 \\
\hline 158 & Deois & pleta & Brasilia (colonial) & 1981 \\
\hline
\end{tabular}




\section{L'isoélectrofocalisation}

Les isoenzymes sont séparées en gels de polyacrylamide à 10 p. 100 contenant 6 p. 100 d'ampholytes ( $(\mathbb{R}$ Pharmalites de PHARMACIA). Les dimensions des plaques de gels sont de $1 \times 115 \times 230 \mathrm{~mm}$. Avant le dépôt de 20 échantillons de $12 \mu \mathrm{l}$ chacun, contenant environ $50 \mu \mathrm{g}$ de protéines, on réalise une préfocalisation pendant $30 \mathrm{mn}$. Les migrations protéiques s'effectuent en $90 \mathrm{mn}$ à $5{ }^{\circ} \mathrm{C}$ sous une puissance constante de $30 \mathrm{~W}$ limitée par une intensité initiale maximale de $100 \mathrm{~mA}$ et une différence de potentiel finale maximale de $2000 \mathrm{~V}$.

Après migration, les gels sont placés en conditions d'incubation caractéristiques de chaque système étudié. Les phosphatases acides (PA) hydrolysent le naphtyl $\alpha$-phosphate en libérant du naphtol détecté en présence de Fast Garnet GBC. Les glutamate-oxaloacétique-transaminases (GOT) sont analysées selon la méthode de NISSELBRAUM \& BODANSKY (1965); les glutamate déshydrogénases (GDH) colorées selon BREWER (BREWER, 1970), tandis que les alcools déshydrogénases $(\mathrm{ADH})$ sont détectées en présence de propanol2 (BREWER, 1970). La technique de AYALA et al. (1972) permet de révéler les malate-déshydrogénases (MDH). Les $\alpha$-estérases sont localisées grâce au naphtyl acétate (TRACEY et al., 1975). Le gradient de pH fut établi empiriquement en fonction de la distribution des isoenzymes de chaque système initialement observé en gamme large de $\mathrm{pH} 3$ à 10 (fig. 1).

\section{E. Mesure de la virulence}

La virulence des souches à l'égard de larves diapausantes du $5^{\text {e }}$ stade de $O$. nubilalis récoltées dans la
Beauce, est estimée par pulvérisation à la tour de traitement (BURGERJON, 1956) de $10 \mathrm{ml}$ d'une suspension à $10^{7} \mathrm{sp} / \mathrm{ml}$. Pour chaque souche 4 lots de 20 larves sont constitués de façon à pouvoir établir un temps létal 50 correspondant à $50 \mathrm{p}$. 100 des larves tuées. Cet insecte fut retenu pour sa sensibilité au germe (RIBA et $a l ., 1983)$ et sa facilité d'élevage.

\section{F. Analyse statistique}

L'analyse factorielle de correspondance (AFC) (CAILLIEZ \& PAGES, 1976) est une méthode d'analyse multifactorielle qui traduit les ressemblances entre individus qui ne sont pas regroupés au départ. Pour chaque souche nous avons codifié arbitrairement chaque isoenzyme par 1 lorsqu'elle est présente et par 0 lorsqu'elle n'est pas synthétisée.

\section{RÉSULTATS}

\section{A. Analyse des zymogrames}

Toutes les souches éprouvées produisent de 5 à $7 \mathrm{~g}$ de culture, à partir desquels on obtient environ $1 \mathrm{ml}$ d'extrait contenant 80 à $100 \mu \mathrm{g}$ de protéines. Les systèmes MDH et GDH comportant respectivement 15 et 1 isoenzymes présentent une remarquable homogénéité au sein de l'échantillon de souches analysées (fig. 1). De même, toutes les souches ont le profil $\mathrm{ADH} \mathrm{E}_{6}$ (fig. 1) à l'exclusion de l'isolat 149 qui produit une isoenzyme supplémentaire (profil $\mathrm{ADH} \mathrm{CM}_{44}$ ). La variabilité de l'échantillon de souches brésiliennes est
Figure 1

Electrophorégrammes enzymatiques obtenus par focalisation isoélectrique au cours de l'analyse de 27 souches brésiliennes de Metarhizium anisopliae. Les chiffres indiquent les valeurs extrêmes $d u$ gradient de $p H$ utilisé. Les lettres signalent les isoenzymes variables. GOT: glutamate oxaloacétique transaminases;

$A D H$ : alcool déshydrogénases ;

$M D H$ : malate déshydrogénases;

$G D H:$ glutamate déshydrogénases.
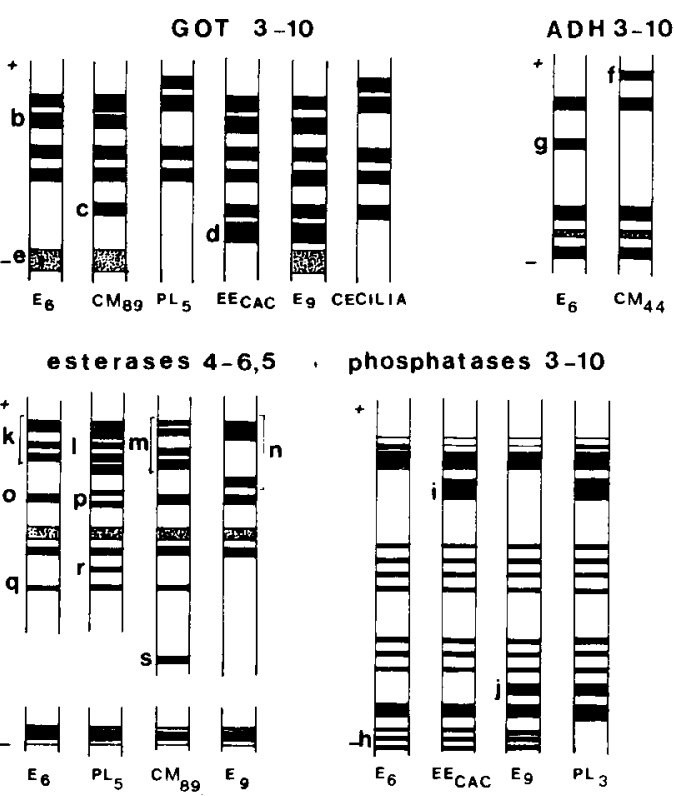

MDH 3-10

GDH 3-10
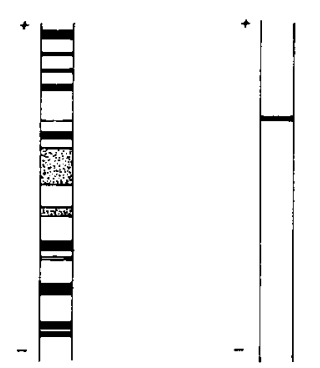

Enzyme electrophoregram obtained by isoelectric focusing of intracellular proteins of 27 Brazilian Metarhizium anisopliae strains. The figures indicate the extreme values of the $\mathrm{pH}$ gradient used; the letters the variable isoenzymes.

GOT : glutamate oxaloacetate transaminases;

$A D H$ : alcohol dehydrogenases;

$M D H$ : malate dehydrogenases;

$G D H:$ glutamate dehydrogenases. 
mieux perçue par l'analyse des 3 autres systèmes éprouvés (tabl. 2 et 3 ). Ainsi le profil phosphatases acides $E_{6}$ est très largement dominant, puisque seules les souches $153,159,171$ et 162 ne le possèdent pas. Finalement, les systèmes GOT et $\alpha$-estérases sont les plus variables pour les 27 souches considérées (tabl. 2). Aucun profil n'est entièrement original pour l'ensemble des enzymes considérées. Cependant on peut remarquer que le type $E_{6}$ diffère de tous les autres par GOT, $\alpha$-estérases et phosphatases acides ; tandis qu'à l'opposé les types $\mathrm{E}_{6}$ et $\mathrm{CM}_{44}$ ne sont séparés que par les $\mathrm{ADH}$.

\section{B. Etude comparative des agressivités}

Il y a une variation continue de l'agressivité des souches à l'égard de larves diapausantes de la pyrale du mais (tabl. 3). Les isolats $150,151,153,155,157,159$ et 171 tuent l'ensemble des larves traitées en moins de

TABLEAU 2

Pouvoir discriminant des systèmes enzymatiques étudiés pour les différents profils électrophorétiques observés au sein d'un échantillon de 27 souches brésiliennes de Metarhizium anisopliae.

Discriminating power of the enzyme systems studied in different electrophoretic profiles observed within a sample of 27 Brazilian Metarhizium anisopliae strains.

\begin{tabular}{|c|c|c|c|c|}
\hline \multicolumn{5}{|c|}{ Systèmes isoenzymatiques } \\
\hline GOT & $\alpha$-estérases & Phosphatases acides & $\mathrm{ADH}$ & $\mathrm{GDH}$ et $\mathrm{MDH}$ \\
\hline $\mathrm{E}_{6}=\mathrm{CM}_{44}$ & $\mathrm{E}_{6}=\mathrm{CM}_{44}$ & $\begin{array}{c}\mathrm{E}_{6}=\mathrm{CM}_{44}=\mathrm{PI}_{55}= \\
=\text { Cécilia }=\mathrm{CM}_{89}\end{array}$ & $\mathrm{CM}_{44}$ & $\begin{array}{l}\mathrm{E}_{6}=\mathrm{CM}_{44}=\mathrm{PL}_{5}= \\
\quad=\mathrm{PL}_{3}=\text { Cécilia }=\end{array}$ \\
\hline $\mathrm{PL}_{5}=\mathrm{PL}_{3}$ & $\begin{aligned} & \mathrm{PL}_{5}=\mathrm{PL}_{3}= \\
= & \mathrm{EE}_{\mathrm{CAC}^{\prime}}=\text { Cécilia }\end{aligned}$ & $\mathrm{PL}_{3}$ & $\begin{aligned} \mathrm{E}_{6}=\mathrm{PL}_{5}=\mathrm{CM}_{89}= \\
=\mathrm{PL}_{3}=\mathrm{EE}_{\mathrm{CAC}}=\mathrm{E}_{9}= \\
=\text { Cécilia }\end{aligned}$ & $=\mathrm{CM}_{89}=\mathrm{EE}_{\mathrm{CAC}}=\mathrm{E}_{9}$ \\
\hline $\begin{array}{l}\mathrm{EE}_{\mathrm{CAC}} \\
\mathrm{E}_{y} \\
\text { Cécilia }\end{array}$ & $\mathrm{E}_{y}$ & $\mathrm{EE}_{\mathrm{CAC}}$ & & \\
\hline $\mathrm{CM}_{89}$ & $\mathrm{CM}_{89}$ & & & \\
\hline
\end{tabular}

\section{TABLEAU 3}

Pathogénic à l'égard des larves de la Pyrale du mais et profils électrophorétiques de souches brésiliennes de Metarhizium anisopliae. Les profils électrophorétiques sont définis par les glutamate-oxaloacétique-transaminases, les phosphatases acides, les $\alpha$-estérases, les alcooldéshydrogénases, les malate-déshydrogénases et les glutamate-déshydrogénases.

Pathogenicity towards Ostrinia nubilalis larvae and electrophoretic profiles of Brazilian Metarhizium anisopliae strains. The electrophoretic profiles are defined by glutamate-oxaloacetate transaminases, acid-phosphatases, $\alpha$-esterases, alcohol-dehydrogenases, malate-dehydrogenases and glutamate-dehydrogenases.

\begin{tabular}{|c|c|c|c|c|}
\hline Souches & TL 50 en jours & Intervalle de confiance & Droite de régression & Profils électrophorétiques \\
\hline 151 & 8,5 & $8,0-9,1$ & $5,6 t-0,3$ & $\mathrm{E}_{6}$ \\
\hline 171 & 9,6 & $9,1-10,1$ & $6,4 t-1,3$ & $\mathrm{E}_{9}^{0}$ \\
\hline 159 & 9,7 & $9,2-10,2$ & $6,31-1,2$ & $\mathrm{E}_{g}$ \\
\hline 150 & 9,7 & $9,2-10,2$ & $6,31-1,2$ & $\mathrm{PL}_{5}$ \\
\hline 155 & 9,7 & $9,2-10,3$ & 51 & $\mathrm{PL}_{5}$ \\
\hline 153 & 9,7 & $9,1-10,3$ & $4,4 t+0,6$ & $\mathrm{EE}_{\mathrm{CAC}}$ \\
\hline 157 & 10,0 & $8,6-11,6$ & $1,8 \mathrm{t}-0,3$ & $\mathrm{E}_{6}$ \\
\hline 164 & 10,6 & $10,1-11,1$ & $6,4 t-1,5$ & $\mathrm{PL}_{5}^{\circ}$ \\
\hline 167 & 10,6 & $10,0-11,2$ & $4,91-0$ & Cecilia \\
\hline 162 & 11,4 & $10,9-12$ & $61-1,3$ & $\mathrm{PL}_{3}$ \\
\hline 166 & 11,7 & $11,1-12,3$ & $5,5 \mathrm{t}-0,8$ & $\mathrm{PL}_{5}$ \\
\hline 163 & 11,8 & $11,2-12,4$ & $5,8 \mathrm{t}-1,2$ & $\mathrm{PL}_{5}$ \\
\hline 169 & 11,8 & $11,3-12,4$ & $6,21-1,7$ & $\mathrm{PL}_{5}$ \\
\hline 149 & 12,1 & $11,6-12,7$ & $6,3+1,9$ & $\mathrm{CM}_{44}$ \\
\hline 158 & 12,8 & $12,3-13,4$ & $6,6 t-2,3$ & $\mathrm{PL}_{5}^{-44}$ \\
\hline 152 & 16,6 & $15,7-17,6$ & $5,5 \mathrm{t}-1,7$ & $E_{6}$ \\
\hline 156 & 16,7 & $15,5-18,0$ & $6 t-2,3$ & $E_{6}$ \\
\hline 170 & 16,8 & $15,3-10,1$ & $4,5 t+0,3$ & $E_{6}$ \\
\hline 154 & 18,6 & $17,1-20,1$ & $4,6 t-0,8$ & $E_{6}^{0}$ \\
\hline 148 & 19,2 & $17,8-20,8$ & $4,3 \div-0,5$ & $E_{6}^{0}$ \\
\hline 147 & 23,9 & $20,4-28,0$ & $2,6 t+1,3$ & $E_{6}$ \\
\hline 161 & 25,5 & $21,9-29,6$ & $4,3 t-1,0$ & $\mathrm{E}_{6}$ \\
\hline 168 & 28,7 & $23,0-35,5$ & $4 i-0,9$ & $\mathrm{PL}_{5}$ \\
\hline 145 & 30,0 & $26,6-34,4$ & $4 t-1,1$ & $\mathrm{E}_{6}$ \\
\hline 146 & 35 & $28,8-42,7$ & $3,2 t+0$ & $\mathrm{CM}_{89}$ \\
\hline 165 & 40,6 & $26-63$ & $2,5 t-0,9$ & $E_{6}$ \\
\hline 144 & 62,3 & $36,5-108,6$ & $2,4 t+0,7$ & $\mathrm{E}_{6}$ \\
\hline
\end{tabular}


12 jours à $25^{\circ} \mathrm{C}$, ce que traduit le faible temps létal 50 qui oscille entre 8 et 10 jours ; mais les souches 144 , 145, 146 et 165 n'affectent la moitié des larves qu'un mois après le traitement. Les 2 souches 159 et 171 de type $\mathrm{E}_{9}$ ont un excellent pouvoir pathogène à l'égard des larves de pyrale du maïs ; par contre, les 2 souches 168 et 150 appartenant toutes deux au type $\mathrm{PL}_{5}$ ont des virulences extrêmement différentes à l'égard de cet insecte (tabl. 3).

\section{Analyse biométrique des données}

L'analyse factorielle de correspondance met parfaitement en valeur les similitudes qui existent entre les 8 types électrophorétiques définis par 19 protéines enzymatiques variables (fig. 2). Certains types, très originaux, ne sont représentés que par une seule souche, telle la souche 149 unique représentante de $\mathrm{CM}_{44}$, ou 146 appartenant au type $\mathrm{CM}_{89}$, ou 167 et 153 respectivement typiques de Cécilia et $\mathrm{EE}_{\text {CAC }}$ (tabl. 3). Certains de ces profils sont parfois caractérisés par quelques isoenzymes particulières telle que la bande $f$ du système $\mathrm{ADH}$ qui est typique du profil $\mathrm{CM}_{44}$; ou les estérases $m$ et $s$ qui ne se trouvent que dans la souche 146. Le type électrophorétique E est caractérisé par les GOT c et d et l'estérase n. Par ailleurs, la projection des diverses caractéristiques biochimiques observées sur le plan 1 (qui représente 88,7 p. 100 de la variabilité totale) confirme les similitudes entre d'une part les types Cécilia et $\mathrm{PL}_{5}$; d'autre part $\mathrm{EE}_{\mathrm{CAC}}$ et $\mathrm{PL}_{3}$; et finalement, $\mathrm{E}_{6}$ et $\mathrm{CM}_{44}$.

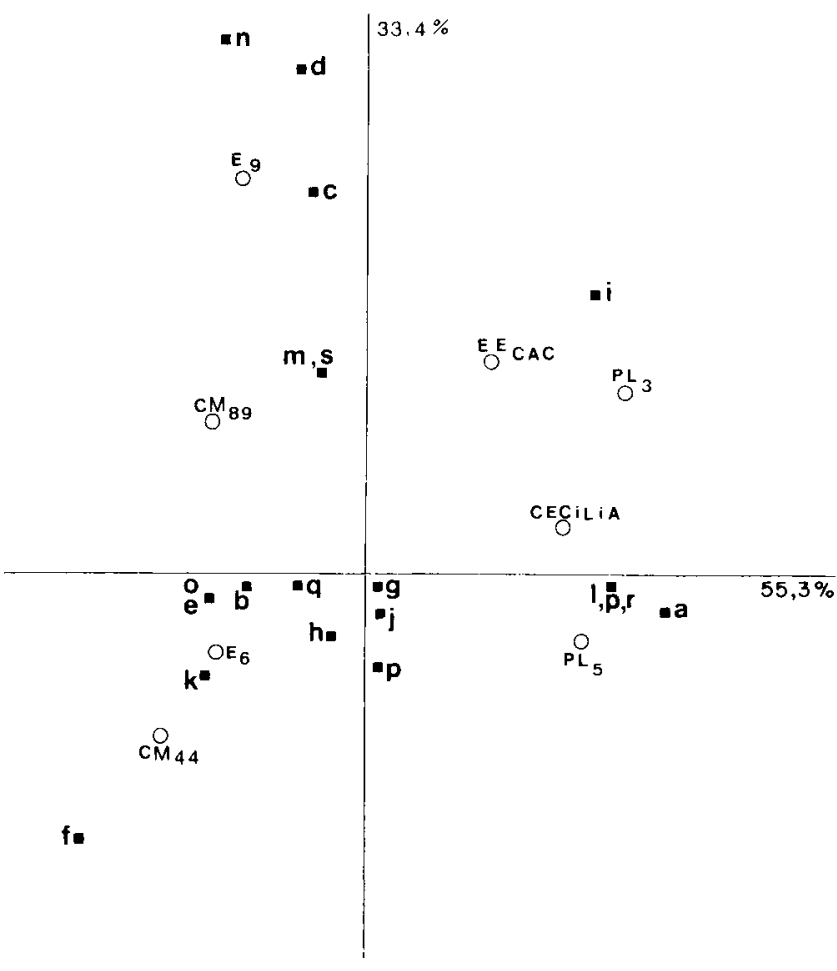

Figure 2

Projection sur le plan principal après analyse factorielle de correspondance des différentes souches brésiliennes de Metarthizium anisopliae isolées de Cercopidae.

Projection on the main plane after factorial correspondence analysis of the different Brazilian strains of Metarhizium anisopliae.

\section{DISCUSSION ET CONCLUSION}

L'analyse électrophorétique de ces échantillons a montré des différences et des ressemblances entre souches d'une même espèce fongique, isolées de la même famille d'insecte-hôte (Cercopidae), dans le même pays. De telles observations complètent celles de DF CONTI et al. (1980) tout en soulignant les avantages et les limites d'une telle approche.

Dans I'espèce $M$. anisopliae, les systèmes enzymatiques choisis sont polymorphes (RIBA et al., 1986). Par conséquent, le fait que les 27 souches analysées possèdent 34 isoenzymes communes est vraisemblablement la résultante des liens phylogénétiques qui unissent tous ces isolats. Cette parenté génétique peut, d'une part s'accompagner d'une cohabitation géographique de souches appartenant à des types électrophorétiques différents ; ainsi des souches de types $\mathrm{E}_{6}$ et $\mathrm{PL}_{5}$ cohabitent dans les champs de l'Usine Triumph (152 et 164) ou dans ceux de l'Usine Saint Michel $(163,165)$; de même à Peixe se trouvent simultanément des souches de type $\mathrm{E}_{6}$ (161) et $\mathrm{PL}_{3}$ (162); d'autre part, il est important de noter que les fréquences de ces divers profils protéiques varient d'une région à l'autre. Ainsi, dans l'état de Pernanbouc les isolats de types $\mathrm{E}_{6}$ (144, $145,147,148$ ) furent plus fréquemment rencontrés que ceux appartenant à d'autres types $(146,149)$. A l'opposé, dans l'état de l'Alagoas les souches de type $\mathrm{PL}_{5}(150,155,163,164,166,168)$ dominent les autres qui se répartissent dans de nombreux profils $(152,156$, $161,162,165,167)$. Cependant, les prélèvements de souches devraient être plus exhaustifs pour être représentatifs de la variabilité et des filiations entre souches sympatriques.

L'observation en un même lieu de génotypes différents de $M$. anisopliae ayant des allèles communs doit être rapprochée de la découverte du cycle parasexuel de ce champignon (AL-AIDROOS, 1980 ; MESSIAS \& DE AZEVEDO, 1980 ; RIBA et al., 1980) conduisant à l'apparition de souches diploïdes ou recombinées aux performances nouvelles (RIBA et al., 1985). Pour l'instant, aucune des enzymes observées ne semble corrélée ou impliquée dans le déterminisme de la virulence de $M$. anisopliae à l'égard de la pyrale du maïs. Ainsi les souches 144,145 et 165 hypoagressives ont les mêmes profils électrophorétiques de la souche 151 dont le temps létal 50 n'est que de 8,5 jours (tabl. 3). Cette absence de corrélation entre la pathogénie et les profils électrophorétiques choisis incite à l'étude du polymorphisme des protéines excrétées par ces germes en diverses conditions de culture.

Dans l'ensemble on peut considérer que le polymorphisme protéique des populations observées est faible. Cette situation peut être la résultante soit d'un nombre d'allèles réduit dans cette région du Nord-Est du Brésil dont le climat est si constant, soit d'une pression de sélection exercée par l'insecte-hôte. Pour estimer le fondement de cette dernière hypothèse, il conviendrait d'étudier le polymorphisme électrophorétique de populations de $M$. anisopliae issues d'autres espèces d'insectes sympatriques de ce Cercopidae. En effet, la spécificité parasitaire des souches de $M$. anisopliae peut se traduire par la sélection par ces insectes de souches génétiquement différentes de celles qui ont été prélevées à partir de cadavres de $M$. posticata (FARGUES, 1976). 
Par ailleurs, et sous réserve que les différentes bandes observées ne résultent pas de variations de charges d'une même isoenzyme, il est généralement admis que la multiplication du nombre des isoenzymes au sein d'organismes asexués serait une compensation de la faible fréquence de remaniements génétiques qu'ils subissent par suite de l'absence de recombinaisons méiotiques (DALMASSO \& BERGE, 1983) et permettrait une meilleure adaptation aux variations de l'environnement (BOUCIAS et al., 1982).

Enfin, l'analyse électrophorétique a permis d'appréhender la stabilité de souches de $M$. anisopliae au cours de cycles de productions industrielles, ou après introduction dans un écosystème.

A ce propos, il est intéressant de noter le maintien des souches 168 ou 154, qui, après deux cycles de production industrielle suivis d'applications dans la nature, ont pu être respectivement réisolées (souches 169 et 170) sans révéler de sensibles modifications des isoenzymes ou de la pathogénie. De même, la souche 151 isolée de Zulia entreriana dans le Mato Grosso a été introduite dans un champ puis prélevée d'un œuf de $M$. posticata (souche 157) sans modification sensible. Par contre la souche 155 qui fut isolée d'une larve de la pyrale Diatraea saccharalis dans un champ préalablement traité avec la souche 151 présente de nombreuses différences avec cette dernière. Il s'agit vrai- semblablement d'une souche autochtone qui par erreur fut assimilée à la souche introduite dont elle ne diffère par aucun caractère morphologique. Cette observation justifie toute la prudence qu'il convient de prendre lors de la réactivation d'une souche à partir d'un insecte prélevé dans la nature et dont on ne connaît pas $a$ priori l'état sanitaire. Les méthodes employées, tout en demandant des approches techniques complémentaires telle que l'immunoélectrophorèse, sont d'un grand intérêt pour le suivi épidémiologique d'opérations de lutte microbiologique.

Malgré ces limites, l'étude du polymorphisme enzymatique de populations d'hyphomycètes est un outil performant qui contribuera à une meilleure compréhension des phénomènes de sélection ou d'hybridation que subissent dans la nature des souches autochtones ou introduites.

Reçu le 18 février 1987. Accepté le 18 juillet 1987.

\section{REMERCIEMENTS}

Nous exprimons nos plus vifs remerciements à M. Helio TeIXeIRA, Chef du Service Fédéral de la Protection des Végétaux pour les nombreuses facilités qu'il a accordées à ce programme, ainsi qu'à M. FILHo, Chef du Service Phytosanitaire de l'Institut de l'Alcool et du Sucre de Carpina pour la fourniture de souches de M. anisopliae.

\section{RÉFÉRENCES BIBLIOGRAPHIQUES}

Al-Aidroos K., 1980. Demonstration of a parasexual cycle in the entomopathogenic fungus Metarhizium anisopliae. Genet. Cytol., $22,771-775$.

Adamek A., 1965. Submerse cultivation of the fungus Metarhizium anisopliae. Folia Microb. (Prague), 4, 255-257.

Ayala F., Powell J., Tracey M., Mourao C., Perez-Salsa S., 1972. Enzyme variability in the Drosophila willistoni group. IV. Genic variation in natural populations of Drosophila willistoni. Genetics, 70, 113-139.

Boucias D. G., McCoy C. W., Joslyn D. J., 1982. Isozyme differentiation among 17 geographical isolates of Hirsutella thompsonii. $J$. Invertebr. Pathol., 39, 329-337.

Brewer G. J., 1970. An introduction to isozyme techniques. Acad. Press., New York, $186 \mathrm{p}$.

Burgerjon A., 1956. Pulvérisation et poudrage au laboratoire des préparations pathogènes insecticides. Ann. Epiph., 4, 677-686.

Cailliez F., Pages J. P., 1976. Introduction à l'analyse des données. Smash ed., $616 \mathrm{p}$.

Dalmasso A., Bergé J. B., 1983. Concepts in Nematode Systematics. In Systematics Associations, Stone A., Platt U. M., Khalil L. F. eds, Acad. Press, 22, 187-196.

De Conti E., Messias C. L., de Souza H. M., Azevedo J. L., 1980. Electrophoretic variation in esterases and phosphatases in eleven wild type strains of Metarhizium anisopliae. Experientia, 36, 293294.

Fargues J., 1976. Spécificité des champignons pathogènes imparfaits (Hyphomycètes) pour les larves de Coléoptères (Scarabeidae et Chrysomelidae). Entomophaga, 21, 313-323.

Guagliumi P., 1970. As cigarrinhas das pastagens e seu controle biologico no nordeste do Brasil. Ruralidade, Goiania, 1, 33-37.

Lowry O. H., Rosebrough N. J., Farr L., Randall R. J., 1951. Protein measurement with the folin phenol reagent. J. Biol. Chem., $193,265-275$.
Messias C., de Azevedo J. L., 1980. Parasexuality in the Deuteromycete Metarhizium anisopliae. Trans. Br. Mycol. Soc., 75, 473-477.

Naves M. A., 1980. As cigarrinhas das pastagens e sugestoes para o seu controle. Contribuçao ao manejo integrado das pragas das pastagens. EMBRAPA-CPAC, Circular tecnica, 3/27 pag.

Naves M. A., Correa H. G., 1982. Aplicaçao aerea de Metarhizium anisopliae (Metsch) Sorok. em pastagens. Anais XII Congresso Brasileiro de Engenharia Agricola CEPLAC/CEPEC - Itabuna - Bahia, 238.

Nisselbaum J. S., Bodansky O., 1965. Glutamic oxaloacetic transaminases in reticulocytes and erythrocytes. Science, 149, 195-107.

Riba G., Glandard A., Ravelojoana A. M., Ferron P., 1980. Isolement de recombinés mitotiques stables de type "intermédiaire" chez Metarhizium anisopliae (Metchnikoff) par hybridation de biotypes sauvages. C. R. Acad. Sci., Paris, Ser. D, 291, 657-660.

Riba G., Marcandier S., Richard G., Larget I., 1983. Sensibilité de la pyrale du maïs (Ostrinia nubilalis) (Lep. Pyralidae) aux Hyphomycètes entomopathogènes. Entomophaga, 28, 55-64.

Riba G., de Azevedo J. L., Messias C., Dias da Silveira W., Tuveson R.. 1985. Studies on the inheritance of virulence in the entomopathogenic fungus Metarhizium anisopliae. J. Invertebr. Pathol., $46,20-25$.

Riba G., Bouvier-Fourcade I., Caudal A., 1986. Isoenzyme polymorphism in Metarhizium anisopliae. Mycopathologia, 96, 161-169.

Tracey M. L., Nelson K., Hedgelock D., Shleser R. A., Pressick M. L., 1975. Biochemical genetics of lobsters - genetic variation and the structure of American lobster (Homarus americanus) populations. J. Fish. Res. Board Canad., 32, 2091-2101.

Veiga A. F. S. L., Aquino M. L. N., Arruda G. P., 1972. Nota sobre o controle biological das cigarrinhas das pastagens (Homoptera, Cercopidae) com fungo entomopathogeno Metarhizium anisopliae (Metsch.) Sorokin no Estado de Pernambuco. Pesquisa Agropec. Nordeste Recife, 1, 71. 